\title{
ZnS/ZnSe SUPERLATTICES UNDER PRESSURE
}

\author{
I. GorCZYCA* AND N.E. ChRISTENSEN \\ Institute of Physics, Aarhus University, 8000 Aarhus C, Denmark
}

\begin{abstract}
Self-consistent linear muffin-tin orbital method is used to calculate the band structure of $\mathrm{ZnS} / \mathrm{ZnSe}(001)$ strained-layer superlattice and investigate the influence of hydrostatic pressure on the valence band offset (VBO). Three different strain modes corresponding to various values of the relative thicknesses of both materials are considered. A I $\rightarrow$ II type conversion associated with the conduction-band crossover between the $\mathrm{ZnSe}$ well and $\mathrm{ZnS}$ barrier layers is found in agreement with recent experimental data.

PACS numbers: 71.25.Tn, 73.20.Dx
\end{abstract}

\section{Introduction}

In a previous paper [1] the electronic-structure calculations for $\mathrm{ZnS} / \mathrm{ZnSe}$ (001) and (110) superlattices were performed, and valence-band offsets (VBOs) were derived. The present work uses the same method, i.e., self-consistent supercell calculations performed within the local-density approximation (LDA) [2], to calculate electronic properties of $\mathrm{ZnS} / \mathrm{ZnSe}(001)$ superlattice as a function of hydrostatic pressure. Three different strain modes are considered: pseudomorphic growth of $\mathrm{ZnSe}$ on a $\mathrm{ZnS}$ substrate, the reverse case, and "free-standing" case (equal thicknesses of both layers).

As already described [1], atomic positions in the interface region are determined by means of valence-force-field calculations in the Keating model [3].

The pressure coefficients of VBOs are found to depend on the strain mode. Generally, the VBO increases with pressure, but whereas this dependence is pronounced in the case where $\mathrm{ZnSe}$ is pseudomorphically grown on a $\mathrm{ZnS}$ substrate it is almost negligible for the reverse case. The more the VBO increases with pressure, the more the conduction band offset (CBO) decreases with pressure. This effect leads to conversion from type I to type II associated with the conduction-band crossover between the $\mathrm{ZnSe}$ well and $\mathrm{ZnS}$ barrier layers. Unfortunately, the LDA causes considerable underestimate of energy gaps, and therefore we have to adjust the gaps to experimental band gaps for bulk materials in order to estimate the CBO. This makes the estimated value of the crossover pressure somewhat ambiguous. Nevertheless, the calculation confirms the experimental finding of the type conversion under hydrostatic pressure [4].

*Also: High Pressure Research Center, Polish Academy of Sciences, Sokołowska 29, 01-142 Warszawa, Poland. 


\section{Method of calculation}

The relativistic linear-muffin-tin-orbital (LMTO) method in the version which applies spherically symmetrized charge distributions and potentials in atomic spheres, i.e. space filling (and thus slightly overlapping) spheres - the atomic-sphere approximation (ASA) [5] — is used to obtain the electronic structure of the considered superlattices. All calculations are performed within the local approximation to the density-functional theory. The $\mathrm{Zn} 3 d$ states are included as fully relaxed band states.

We consider three different strain modes corresponding to various values of the relative thicknesses of both materials:

$A$. the lattice constant parallel to the interface is that of bulk $\mathrm{ZnS}$, i.e. corresponding to a pseudomorphic growth of $\mathrm{ZnSe}$ on a $\mathrm{ZnS}$ substrate;

$B$. "free-standing" case - ZnS, as well as ZnSe layers, both with the same thickness, assume lattice constants different from the bulk values [1],

$C$. pseudomorphic growth of $\mathrm{ZnS}$ on a $\mathrm{ZnSe}$ substrate;

Results for the (001) $\mathrm{ZnS} / \mathrm{ZnSe}$ superlattices at zero pressure were presented in the previous paper [1]. Here we focus our attention on the pressure effects.

\section{ZnS/ZnSe superlattice under pressure}

The electronic band structure and total energy calculations were performed first for $\mathrm{ZnS}$ and for $\mathrm{ZnSe}$ bulk materials. From these calculations we obtained the volume-pressure relations, the equilibrium lattice constants and the bulk moduli. The obtained values of bulk moduli are starting points for the calculations of the electronic structure of superlattice for different values of pressure. Here we need the

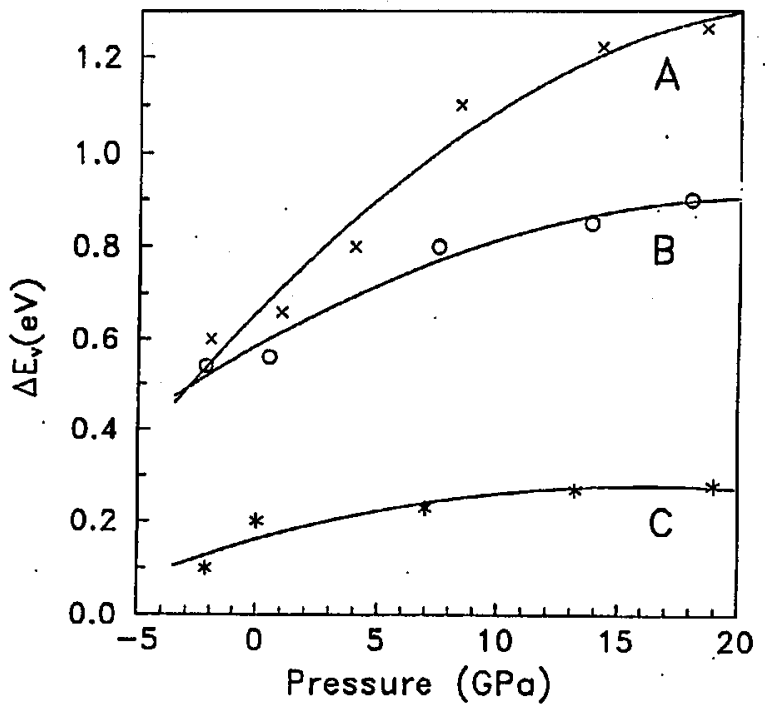

Fig. 1. Pressure dependence of the valence band offset for $\mathrm{ZnS} / \mathrm{ZnSe}$ superlattice. 
values of elastic constants $c_{11}(p)$ and $c_{12}(p)$ for both sides of heterostructure. We take the values which correspond to our calculated bulk moduli $B_{0}(p)$, assuming experimental values $[6,7]$ of the ratios $c_{11} / c_{12}$ and experimental values $[6,7]$ of the ratios $\left(\mathrm{d} c_{11} / \mathrm{d} p\right) /\left(\mathrm{d} c_{12} / \mathrm{d} p\right)$.

In order to study the pressure dependence of the electronic structure of $\mathrm{ZnS} / \mathrm{ZnSe}$ superlattice the band structure and total energy calculations were performed for some values of hydrostatic pressure. From the total energy dependence on volume we obtained the volume-pressure relation, the equilibrium average lattice constants, the bulk moduli, and their pressure coefficients. From the pressure dependence of the electronic band structure we deduced the pressure dependence of the valence band offset, $\Delta E_{\mathrm{v}}$, defined as the difference between the energies of the highest occupied states in $\mathrm{ZnSe}$ and $\mathrm{ZnS}$. The calculated VBOs are very sensitive to the strain conditions. As we can see from Fig. 1, the VBO increases rapidly with pressure for the strain mode $A$, slightly less for $B$ and it is almost constant for mode $C$. The pressure behavior of VBOs is strongly nonlinear and about $15 \mathrm{GPa}$ (region of phase transitions in $\mathrm{ZnS}$ and $\mathrm{ZnSe}$ ) they become almost pressure independent.

The values of valence-band deformation potentials previously obtained [1] for $\mathrm{ZnS}$ and $\mathrm{ZnSe}$ by the strained homojunction method are: $-3.5 \mathrm{eV}$ and $-1.2 \mathrm{eV}$, respectively, therefore in the absence of internal strain and neglecting interface effects VBO should decrease with pressure. Thus, the increase in the VBO with pressure cannot be explained in terms of the deformation potentials for bulk materials - built-in strain plays a decisive role here.



Fig. 2. Pressure dependence of the conduction-band edges for both $\mathrm{ZnS}$ and $\mathrm{ZnSe}$ layers for strain mode $A$, i.e., pseudomorphic growth of $\mathrm{ZnSe}$ on a $\mathrm{ZnS}$ substrate. The vertical dashed line indicates the crossover pressure. 
In agreement with experiment [4] we find that the conduction band offset at zero pressure has the opposite sign of the VBO, i.e. the superlattice is of type I. According to the recent experimental data [4] a conversion from type I to type II of the superlattice is observed at about $3 \mathrm{GPa}$. We also observe the $\Gamma-\Gamma$ conduction band crossover between the $\mathrm{ZnSe}$ well and $\mathrm{ZnS}$ barrier layers for $A$ case. To perform more detailed comparison with experiment [4] we have, at first, to adjust the obtained values of the energy gaps to their experimental values for zero pressure (LDA, as it is well known, underestimates considerably values of energy gaps) taking into account strain effects caused by lattice nonmatching. To this purpose we use the deformation potentials of the energy gaps obtained for the bulk materials. Next, we determine the conduction band offset, $\Delta E_{\mathrm{c}}$, at zero pressure as: $\Delta E_{\mathrm{c}}=E_{\mathrm{g}}(\mathrm{ZnS})-E_{\mathrm{g}}(\mathrm{ZnSe})-\Delta E_{\mathrm{v}}$. The resulting pressure dependence of the conduction band edges for the strain mode $A$ is presented in Fig. 2. Our value of critical pressure $P_{\mathrm{c}}$ for conduction band crossover is about $4.5 \mathrm{GPa}$, somewhat more than experimental value $3 \mathrm{GPa}$ [4] (situation described in Ref. [4] is close to our case $A$, since the $\mathrm{ZnS}$ layers are twice as thick as the $\mathrm{ZnSe}$ layers), but we consider our value as a rough estimate due to uncertainty in the energy gaps values at zero pressure.

In conclusion, the self-consistent supercell calculation shows that the valence band offset depends on hydrostatic pressure and is strongly affected by the internal strain. Our calculation confirms the experimental observation of a pressure induced type conversion in $\mathrm{ZnS} / \mathrm{ZnSe}$ superlattice.

\section{References}

[1] N.E. Christensen, I. Gorczyca, Phys. Rev. B 44, 1707 (1991).

[2] P. Hohenberg, W. Kohn, Phys. Rev. 136, B964 (1964); W. Kohn, L.J. Sham, ibid. 140, A1133 (1965).

[3] P.N. Keating, Phys. Rev. 145, 637 (1966).

[4] Y. Yamada, Y. Masumoto, T. Taguchi, K. Takemura, Phys. Rev. B 44, 1801 (1991).

[5] O.K. Andersen, Phys. Rev. B 12, 3060 (1975).

[6] J.C. Jamieson, H.H. Demarest, J. Phys. Chem. Solids 41, 963 (1980).

[7] B.H. Lee, J. Appl. Phys. 41, 2984 (1970). 